\title{
Stolpersteine in der beruflichen Wiedereingliederung
}

Bruno Soltermann

Präsident Swiss Insurance Medicine SIM
Korrespondenz:

Dr. med. Bruno Soltermann Facharzt für Chirurgie FMH C.F. Meyer-Strasse 14 $\mathrm{CH}-8022$ Zürich Tel. 0442082865 Fax 0442082835
Die Swiss Insurance Medicine SIM hat die diesjährige Tagung vom 24. März 2011 unter das Motto «Stolpersteine in der beruflichen Wiedereingliederung» gestellt.

Arbeit betrifft uns alle, das Individuum, die Gesellschaft und die Wirtschaft, die Gesundheit ebenso. Langzeitausfälle bei der Arbeit im Zusammenhang mit gesundheitlichen Problemen kosten die schweizerische Wirtschaft sowie das soziale und private Versicherungssystem immer mehr. Dies führt zu steigenden Belastungen der Privathaushalte durch Steuern und Prämien. Die Arbeitslosen selber haben nicht nur finanziellen Schaden, sondern verlieren rasch ihre Rolle in Gesellschaft und Familie, und die daraus resultierenden schlechteren Lebensperspektiven wirken krankmachend. Als prognostisch grösstes Risiko für eine Langzeitabsenz respektive ein definitives Ausscheiden aus dem Arbeitsprozess und damit auch für eine Berentung wirken die Dauer der Arbeitsunfähigkeit und gehäufte Arbeitsausfälle.

In den letzten Jahren wurden verschiedene Massnahmen ergriffen, um die Dauer einer Arbeitsabsenz möglichst kurz zu halten und so die Abwärtsspirale rechtzeitig zu unterbinden. Als Akteure in der beruflichen Eingliederung wirken Arbeitgeber, ärztliche Grundversorger, zunehmend auch in Form von Ärztenetzwerken, Rehabilitationszentren, Case Manager, Berufsberater und Arbeitsvermittler, die Invalidenversicherung, die Regionalen Arbeitsvermittlungen, die Unfallversicherer wie auch Krankentaggeldversicherer und Lebensversicherer mit der Unterstützung von Versicherungsmedizinern. Andere wie z.B. die Krankenversicherer wirken indirekt auf die Eingliederung. Trotz vieler Bemühungen und zuletzt positiven Entwicklungen in Bezug auf Neuberentungen in der IV bestehen Stolpersteine bei der beruflichen Wiedereingliederung. Einige wurden an der Tagung aufgezeigt und diskutiert: ein kompliziertes Sozial- und Versicherungssystem mit verschiedenen, zum Teil divergierenden Interessenlagen, zu lange Kommunikationswege, Rollenkonflikte bei Ärzten, ein periodischer Krankheitsverlauf, Kostenbremse bei der Krankenversicherung und beim Staat, wenig Tradition in der Gesundheitsvorsorge am Arbeitsplatz, Datenschutz, Eingliederungsziele bei gleichzeitigen Sparzielen in der IV, Vorhandensein geeigneter Arbeitsplätze und steigender Produktivitätsanspruch in der Arbeitswelt.

Initiativen kommen von den Arbeitgebern, von medizinischer Seite und von den Versicherern. Alle waren sich trotz unterschiedlicher Interessenlage einig, dass Lösungsansätze auf der Erkenntnis beruhen müs- sen, dass die berufliche Reintegration kranker oder verunfallter Menschen dann erfolgversprechend ist, wenn sie früh beginnt, fachkompetent betrieben wird und koordiniert erfolgt. Unbeantwortet bleiben die Fragen, wie diese mit Engagement betriebenen institutionsspezifischen Ansätze in einen gemeinsamen, übergreifenden Lösungsansatz überführt werden können und wer diesen Versuch zur gemeinsamen übergreifenden Lösung anführt. Letztlich wird die Politik eine wichtige Rolle bei der Gestaltung der Rahmenbedingungen spielen.

Die SIM möchte darauf hinweisen, dass die Ärzteschaft bei der beruflichen Wiedereingliederung wohl die wichtigste Rolle spielt. Die Bestrebungen, Patientinnen und Patienten wieder an den Arbeitsplatz zurückzuführen, muss nicht gratis geschehen, wie dies leider immer wieder zu hören ist. Für die IV gilt bereits seit April 2008 ausserhalb TARMED eine Stundenlohnentschädigung für Ärztinnen und Ärzte, die sich in der Eingliederung für ihre Patienten engagieren. Eine Besprechung zwischen Arzt, Versicherten und IV-Stellenmitarbeitern kann mit dem Leistungscode 299 «Andere Abklärungen» verrechnet werden. Hier gilt der Ansatz von 200 Franken pro Stunde. Die Suva hat solche Abgeltungen innerhalb verschiedener Ärztenetzwerke vertraglich abgeschlossen, und die privaten Unfallversicherer werden in Kürze diese Honorierungen ausserhalb TARMED für die gesamte Ärzteschaft einführen, die Ansätze sind ebenfalls 200 Franken pro Stunde.

\section{Versicherungsmedizinisches Forum Risiko- prüfung in der Personenversicherung}

Die Swiss Insurance Medicine SIM hat zusammen mit Fachpersonen im Lebensversicherungsbereich der Privatversicherer ein Grundmodul Risikoprüfung in der Lebensversicherung erarbeitet und am 27. Januar 2011 bereits zum dritten Mal durchgeführt. Die SIM möchte nun einen Schritt weiter gehen und ein versicherungsmedizinisches Forum unter den Aspekten der Risikoprüfung in der Personenversicherung durchführen. Das Thema widmet sich jeweils dem vorangegangenen halbjährlich erscheinenden Medinfo des Schweizerischen Versicherungsverbandes, und die Thematik innerhalb des Forums wird dann vertieft. Das Medinfo wird mit der SÄZ an alle Ärzte verteilt, einsehbar auch unter www.svv.ch/publikationen.

Das erste versicherungsmedizinische Forum widmet sich dem metabolischen Syndrom. Es wird am Donnerstag, 19. Mai 2011 von 13.30-17.30 Uhr im Auditorium Tüfihaus, SwissRe, Adliswil durchgeführt. Anmeldung unter www.swiss-insurance-medicine.ch 